\title{
Execution redundancy: the effect of varying technique for a similar outcome on player's groundstroke performance
}

\author{
Michael Davis Higuera ${ }^{a}$ \\ ${ }^{a}$ Tennis Development, Integrity and Development Department, International Tennis Federation, London, \\ UK.
}

\section{ABSTRACT}

Execution redundancy, defined as varying technique for a similar outcome, is a newer defined type of variability which opens new avenues of thinking about tennis training. 19 club-level tennis players were put through different training interventions: low variability where players had to rally to hit a target and maintain technique; and, high variability where players did the same but varied technique slightly. The high variability group improved after a retention period of 1 week in a test of accuracy (measuring average distance of 4 balls hit to a target) and success (measuring number of balls over the net and within a target radius of $500 \mathrm{~cm}$ ). The results suggest that that varying technique for a similar outcome improves performance more than low variability, possibly due mechanisms surrounding increased exploration creating better adapted motor patterns. The results show positive support for variability of this kind of training, but care needs to be taken to ensure that sound biomechanical and technical principles are observed.

\author{
Key words: Variability, \\ execution redundancy, varying \\ technique, methodology. \\ Received: 16 August 2019 \\ Accepted: 01 October 2019 \\ Corresponding author: Michael \\ Davis Higuera, ITF Bank Lane, \\ Roehampton, London, \\ SW5XYZ, United Kingdom. \\ Email: \\ mdavis.higuera@yahoo.com
}

\section{INTRODUCTION}

Variability should be an essential part of every coaches toolkit, present within every teaching methodology in different ways, such as: discovery learning, by allowing the player to explore different solutions to solve a problem (Crespo and Miley, 1998); in variable practice - varying the different situations of practice (the incoming ball) (Pankhurst, 2013); in varied practice - varying the outcome of the shot (Pankhurst, 2013) ; or even, in distributed or random practice (Shea and Morgan, 1979; Reid et al., 2006) - varying the order of practices and intertwining learning of one skill with others.

Even with a beginner trying to adhere to a very strict technical model, variability is present as the learner is exploring motor solutions to try to replicate the skill and outcome. In the same way, a high-performance player trying to hone a skill performs many slightly varying repetitions in order to improve the outcome. However, this does not mean that high amounts of variability should always be prescribed. A good coach is one who is able to decide which different learning styles and coaching methodologies are suitable for each player in each situation (Crespo and Miley, 1998). Thus it is the coach's duty to understand that variability is present in the learning process in one way or the other as well as decide how to best prescribe this variability.

The mechanisms of why variability may lead to better learning have been put down to explanations of: contextual interference - constant forgetting and recall of different skills or variations of skills in a random order facilitating improved consolidation (Shea and Morgan, 1979) through deeper perceptual trace (Adams, 1971); schema theory - variation and repetition adding to a general motor programme through schema (or rules) (Schmidt, 1975); noise and stochastic resonance (Schollhorn et al., 2006) - the clashing of brain signals caused by internal movement and external situations causing the player's movement dynamics to move out of dips of complacency in order to find better solutions; and, dynamic systems (Davids et al., 2008) - where exploration of the movement-skill-outcome landscape (made up of individual, task and environmental constraints) leads to increased ability to perform successful skills more adapted to the individual's capacities and predispositions. 
According to the aforementioned theories, the final two especially (Davids et al., 2008; Schoolhorn et al., 2006), the mechanism behind learning may be non-linear, which is to say that because of the confluence of many different factors and degrees of freedom, training one aspect may lead to a change in an unrelated aspect. However, the theory of specificity of practice suggests that the best training for a test occurs when the training is the same as the test (Shea and Kohl, 1990). In terms of variability, this would imply that to best improve the ability to vary in a certain way, i.e. different shot placement, switching between tasks, or hitting from different situations, then practice should occur in the same way.

One less-studied category of variability is that of that is varying technique whilst maintaining a similar outcome, which could be termed execution redundancy (Ranganathan and Newell, 2010; 2013). This type of variability contrasts with task-goal (structured or unstructured) variability (Ranganathan and Newell, 2010) - varied, variable or even random practice.

If the specificity of practice theory is believed to be absolute in the mechanism behind this type of variability, changing technique in small ways for a similar outcome would only improve performance where this type of variability was required, i.e. where changing technique was important. It might seem counterintuitive to train this type of variability as it stands to reason that if a specific outcome is desired, then the skill should be executed in the same way; however, in tennis, top players respond to hopeless situations with winning and unexpected shots. Thus, this type of practice would benefit performance where a player is under time or space pressure/constraints and must adapt their technique in order to succeed.

If the specificity of practice theory is not believed to be absolute, then execution redundancy, or changing technique slightly for a similar outcome, might still facilitate improved learning and training owing from other aforementioned mechanisms of variability (Schmidt, 1975; Shea and Morgan, 1979, Schollhorn et al., 2006; Davids et al., 2008).

Thus, the aims of this study were to investigate whether this type of variability has an effect on training groundstrokes and if so, explore by what mechanisms this effect takes place.

\section{METHODS}

The study was conducted as a part of a dissertation in support for a degree award (Davis Higuera, 2018). This experiment aimed to study the effect that asking a group of intermediate/advanced players to vary technique in training for the same outcome had on their stroke effectiveness. Two tests were designed and were carried out to test whether this type of variability fell prey to the specificity of practice theory.

19 club-level advanced recreational players (mean age $=46$ years, S.D. = 13 years) gave informed consent for the study and were randomly assigned into two groups, a highvariability experimental group $(n=9)$ and a low-variability control group ( $n=11$ ). Each group underwent a different practice condition with a pre-test immediately before, immediately after and a week later, for two different types of tests. Shot accuracy and shot success were taken as the main variable dependent variables, and were measured in both tests from 4 shots each test: for shot success by counting how many shots of four shots fed to the player were hit into the court and an area with a $500 \mathrm{~cm}$ radius from a target; and, for shot accuracy by measuring the average distance of the landing position from the four shots, fed to the player, from a centre mark on the other side of the court (with a maximum score of $500 \mathrm{~cm}$ for shots further than this distance and missed shots).

The two tests were as follows: 1) a lower pressure test where a ball machine fed four shots down the middle of the court alternating to the forehand and the backhand slightly of the player standing on the centre mark (starting on the right/deuce side) - to test whether high-variability or execution redundancy would positively affect learning even though the situation does not explicitly require variability/adaptation; and, 2) a higher pressure test as above with four shots but feeding wider more angled shots - to test whether high-variability would cause improvements in a pressured condition where adaptation and variability may be more required, as suggested by the specificity of practice theory.

For the intervention in each group, players were asked to rally in pairs (and a three) from the baseline in a straight line with forehand and backhand topspin strokes for $\mathbf{4 0}$ minutes, whilst trying to hit a target approximately halfway between the baseline and service line. The high-variability experimental group was given instructions to make small changes to their technique by exploring slightly different arm configurations through their strokes (changing technique constantly from shot to shot): different degrees of flexion of the elbow and wrist, different follow through positions, and different contact positions relative to the body. The group was monitored and directed by a coach to vary technique stroke to stroke, maintain a topspin rally ball, but still respect effective proven biomechanical and technical principles. The low-variability was not given instructions to vary technique but was still directed to hit a topspin rally ball and maintain solid biomechanical and technical principles.

A two-way repeated measures ANOVA was used to analyse the data for both groups for each test, and the results were analysed using SPSS v.24. The study was approved by the ethics board at Manchester Metropolitan University.

RESULTS

Results are shown for each of the two dependent variables.

Shot success

Figure 1 shows the shot accuracy as measured by the average number of shots (of 4) that were hit over and in, and within $500 \mathrm{~cm}$ of the target, for both tests. 


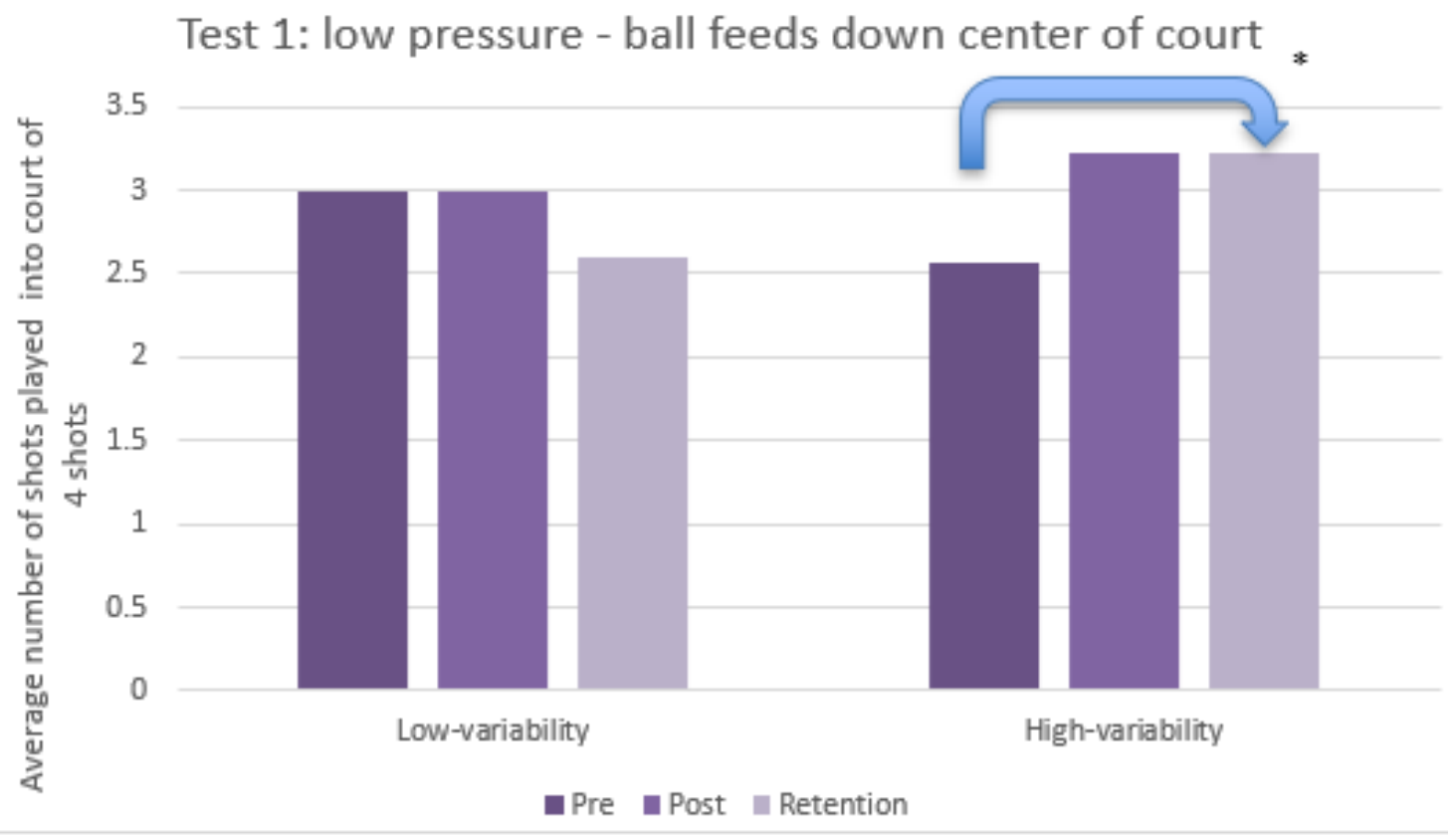

Test 2: high pressure - ball feeds wide

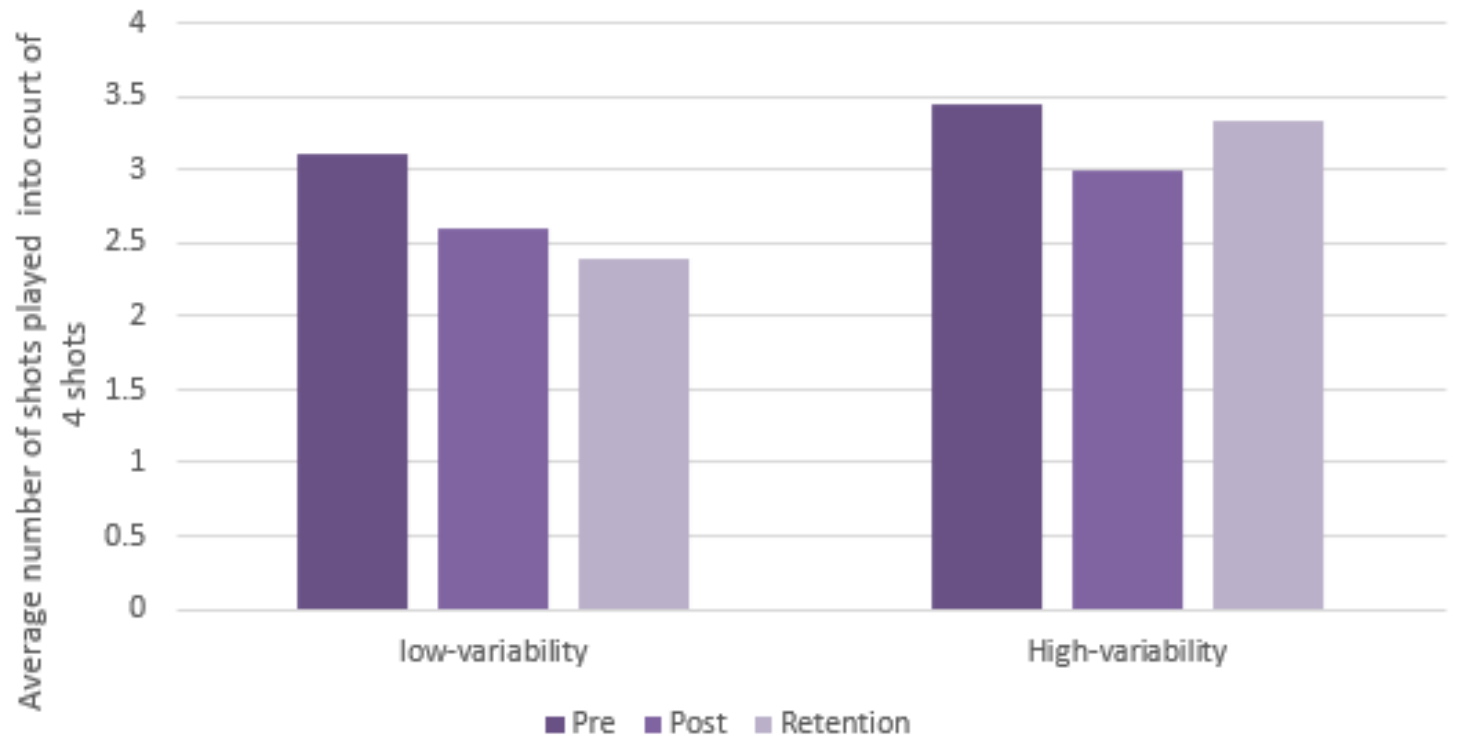

Figure 1. Shot success (average number of shots played in court of 4 shots) for both tests and groups, pre-, post-and 1-week-postintervention. *Bonferroni post-hoc analysis shows a significant increase in number of balls played into the court of $0.667, p=0.044$, is shown for the high-variability group in test 1 between the pre-test and retention-test only.

\section{Shot accuracy}

Figure 2 shows the shot accuracy as measured by the average distance of the 4 shots per player from a target, where missed shots and shots further than a radius of $500 \mathrm{~cm}$ from the target counted as $500 \mathrm{~cm}$, for both tests. 
Test 1: low pressure - ball feeds down center of court
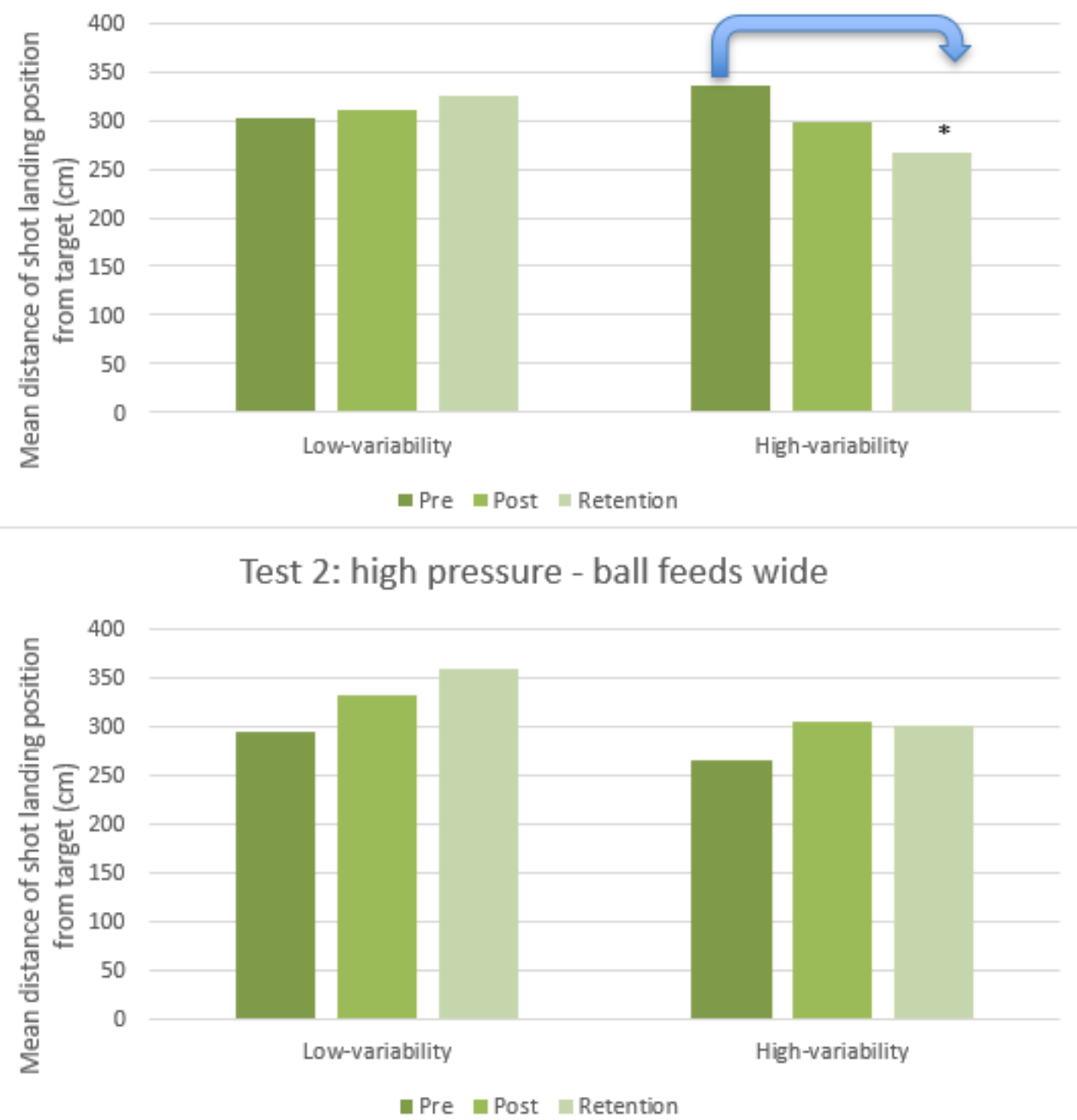

Figure 2. Shot accuracy (average distance form target of shots played into the court of 4 shots with a max. of $500 \mathrm{~cm}$ for missed shots and shots further than $500 \mathrm{~cm}$ ) for both tests and groups, pre-, post-and 1-week-post-intervention. A significant decrease in distance (increase in accuracy) of $68.69 \mathrm{~cm}, p=0.038$, is shown for the differential group in test 1 between the pre-test and retention-test only.

\section{DISCUSSION}

The results show that training with high variability in execution redundancy has a positive effect on shot accuracy and success, but only in the lower pressure test which suggests that high-variability, and prescribing variability in technique, even for a similar outcome, improves performance. The results do not fully support the specificity of practice theory since the practice of varying technique for a similar outcome should best improve performance in a test where this is required; however, the benefits were not isolated to that test. This means that benefits may be due to a more general mechanism of variable simply stimulating and enhancing the learning process. According to the results, this improvement occurs at retention level only which suggests that time is needed for consolidation, i.e. for the benefits to manifest neurologically.

Accuracy and shot success were the variables measured, and together they translate to shot effectiveness. Shot efficiency was not measured although it may be possible to imply that with increased effectiveness, there may be improved efficiency. What might also be possible to state is that due to the increased effectiveness, the individual has found motor solutions, or variation of solution, which are better suited to the player's capacities resulting in an ability to hit the shots with more effectiveness. 
The possible mechanism of improvement is probably explained by a synthesis of the aforementioned theories related to: the shifting of movement dynamics out of complacency (Schollhorn et al., 2006); the player exploring movement patterns more suited to individual capacities (Davids et al., 2008); and, a better developed general motor pattern (Schmidt, 1975).

\section{CONCLUSION}

The results suggest that asking players to vary technique slightly for similar outcomes can speed up learning. However, It is important that this type of coaching still follows other well-established coaching principles, sound biomechanical and sound technical principles in order to maximise efficiency and effectiveness.

This type of training might be best reserved for more advanced players. For intermediate players in the associative stage of learning, a coach might show the player the technique, give the player a range of movement which could be acceptable, and then ask them to explore within that range; although, with a total beginner, the player's action is likely to already be variable and so extra variability should not be prescribed. As always, the skill in coaching lies in knowing what the player needs to progress.

With more effective learning, skills potentially more attuned to the player's capacities and players with more autonomy, the potential for more motivated, individual, well-rounded players increases. Many coaches, possibly the best ones, are already using this (probably without knowing it) by not being too prescriptive, encouraging some flexibility and giving players acceptable ranges to work within rather than fixed and inflexible standards/norms.

Although promising, research surrounding specific interventions of variability in tennis is still in its infancy, especially in the context of varying technique, and so the field should be explored further with different populations and under different conditions.

\section{REFERENCES}

Crespo, M. and Miley, D. (1998). ITF Advanced Coaches Manual. ITF: London

Davids, K., Button, C. \& Bennett, S. (2008) Dynamics of skill acquisition: a constraints-led approach. Leeds; Champaign, II: Human Kinetics.

Davis Higuera, M. (2018). execution redundancy variability of practice: effects of high execution redundancy on recreational tennis player's forehand accuracy (Unpublished dissertation). Manchester Metropolitan University, United KingdomSahan, A., Erman, K.A. \& Ertekin, E. (2018). The effect of a variable practice method on tennis groundstroke learning of adult beginners. ITF Coaching and Sport Science Review, 74(26), 15 - 17.
Pankhurst, A. (2013). How tennis players learn motor skills: Some considerations. ITF Coaching and Sports Science Review, 60(21), pp. 6-7.

Ranganathan, R. \& Newell, K. (2010) "Motor learning through induced variability at the task goal and execution redundancy levels", Journal of motor behaviour, 42(5) pp. 307-316, https://doi.org/10.1080/00222895.2010.510542

Ranganathan, R. \& Newell, K.M., (2013). 'Changing up the routine: intervention-induced variability in motor learning.' Exercise and sport sciences reviews, 41(1), pp.64-70, https://doi.org/10.1097/JES.0b013e318259beb5

Reid, M., Crespo, M., Lay, B. \& Berry, J. (2006). Skill acquisition in tennis: Research and current practice. Journal of Science and Medicine in Sport, 10(1), 1-10, https://doi.org/10.1016/i.jsams.2006.05.011

Sahan, A., Erman, K.A. \& Ertekin, E. (2018). The effect of a variable practice method on tennis groundstroke learning of adult beginners. ITF Coaching and Sport Science Review, 74(26), 15 - 17.

Schmidt, R. A. (1975). 'A schema theory of discrete motor skill learning.' Psychological review, 82(4) pp. 225-260, https://doi.org/10.1037/h0076770

Schollhorn, W. I., Beckmann, H., Michelbrink, M., Sechelmann, M., Trockel, M. \& Davids, K. (2006). Does noise provide a basis for the unification of motor learning theories? International journal of sport psychology, 37(23) pp. 186206.

Shea, C. \& Kohl, R. (1990). Specificity and Variability of Practice. Research Quarterly for Exercise and Sport, 61(2), 169-177, https://doi.org/10.1080/02701367.1990.10608671

Shea, J. B. \& Morgan, R. L. (1979) 'Contextual interference effects on the acquisition, retention, and transfer of a motor skill.' Journal of Experimental Psychology: Human Learning and Memory, 5(2) pp. 179-187, https://doi.org/10.1037/0278-7393.5.2.179

\section{RECOMMENDED ITF TENNIS ACADEMY CONTENT (CLICK BELOW)}

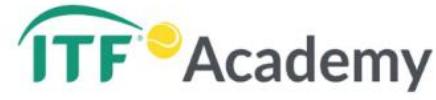

Copyright (c) 2019 Michael Davis Higuera

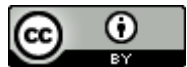

This text is under a Creative Commons BY 4.0 license

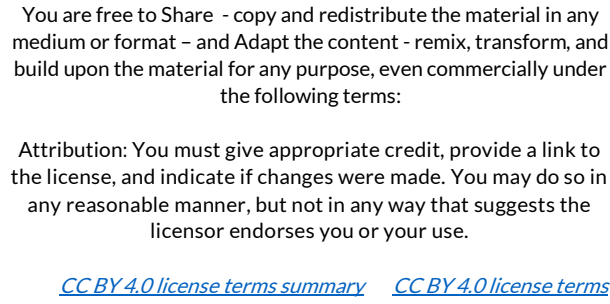

You are free to Share - copy and redistribute the material in any medium or format - and Adapt the content - remix, transform, and build upon the material for any purpose, even commercially under
the following terms:

Attribution: You must give appropriate credit, provide a link to the license, and indicate if changes were made. You may do so in licensor endorses you or your use.

CCBY 4.0license terms summary $\underline{C C B Y 4.0 \text { license terms }}$ 